\title{
Antiferromagnetism and its origin in iron-based superconductors
}

\section{(Review Article)}

\author{
Ming-Cui Ding ${ }^{1}$, Hai-Qing Lin $^{2}$, and Yu-Zhong Zhang ${ }^{1}$ \\ ${ }^{1}$ Shanghai Key Laboratory of Special Artificial Microstructure Materials and Technology, School of Physics Science \\ and Engineering, Tongji University, Shanghai 200092, P.R. China \\ E-mail: yzzhang@tongji.edu.cn \\ ${ }^{2}$ Beijing Computational Science Research Center, Beijing 100084, China
}

Received August 26, 2013

In iron-based superconductors, unravelling the origin of the antiferromagnetism is a crucial step towards understanding the high- $T_{C}$ superconductivity as it is widely believed that the magnetic fluctuations play important roles in the formation of the Cooper pairs. Therefore, in this paper, we will briefly review experimental results related to the antiferromagnetic state in iron-based superconductors and focus on a review of the theoretical investigations which show applicability of the itinerant scenario to the observed antiferromagnetism and corresponding phase transitions in various families of the iron-based superconductors. A proposal of coupling between frustrated and unfrustrated bands for understanding the reduced magnetic moment typically observed in iron pnictides is also reviewed. While all the above theoretical investigations do not rule out a possible existence of localized electrons in iron-based superconductors, these results strongly indicate a close relation between itinerant electrons and the magnetically ordered state and point out the importance of taking into account the orbital degrees of freedom.

PACS: 74.70.Xa Pnictides and chalcogenides;

75.10.-b General theory and models of magnetic ordering;

71.20.-b Electron density of states and band structure of crystalline solids;

71.10.Fd Lattice fermion models (Hubbard model, etc.).

Keywords: high- $T_{c}$ superconductivity, iron-based superconductors, bicollinear antiferromagnetism.

\section{Contents}

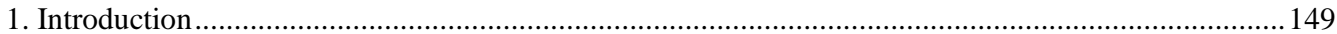

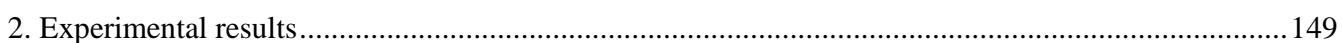

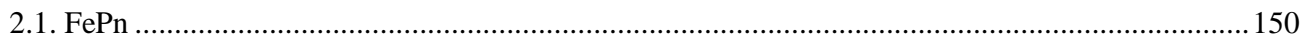

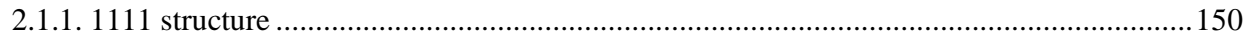

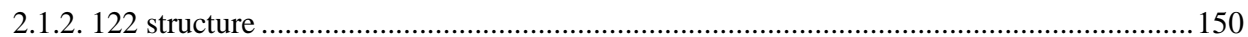

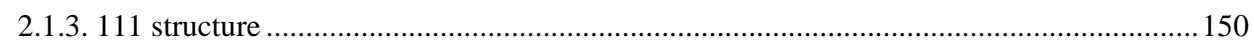

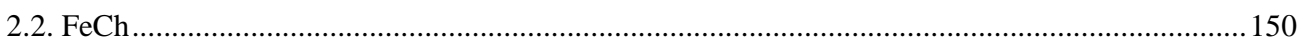

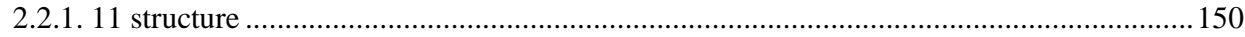

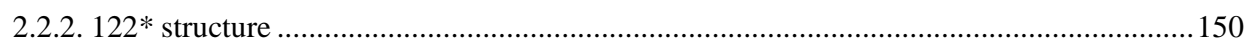

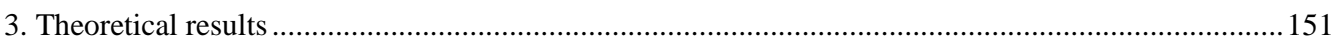

3.1. Origin of reduced magnetic moment ..................................................................................... 151

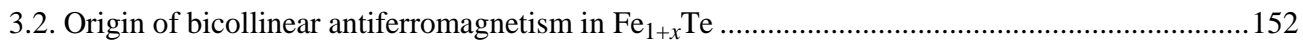

3.3. Origin of magnetic phase transitions in 122 compounds.......................................................... 154

3.4. Origin of collinear antiferromagnetic states in 111 and 1111 families ........................................155

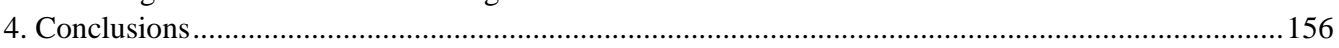

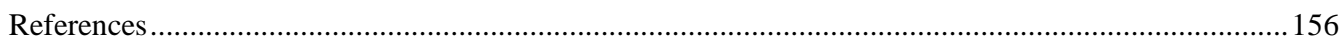




\section{Introduction}

Since the first high- $T_{C}$ iron-based superconductor was discovered in 2008, rapid progress has been achieved in this research field. While the measured transition temperature has been rapidly raised from $26 \mathrm{~K}$ [1] in LaOFeAs to $55 \mathrm{~K}$ as $\mathrm{La}$ is replaced by $\mathrm{Sm}$ [2], it was reported from Xue's group et al. [3] that there may be significantly large superconducting gap in the single-layer FeSe grown on $\mathrm{SrTiO}_{3}$ substrate, which may open a possible avenue to synthesize new superconductors with higher transition temperature.

Apart from great experimental effort in seeking new iron-based superconductors with transition temperature higher than liquid nitrogen [4-18], much attention also has been paid to theoretical understanding of the origin of the high- $T_{C}$ superconductivity [19-26]. It is now generally believed that the antiferromagnetic fluctuations must play an important role in the formation of Cooper pairs when the long range antiferromagnetic order is suppressed. This is due to the fact that the electron-phonon coupling alone cannot account for the high transition temperature observed experimentally and the superconducting phase usually appears in the vicinity of antiferromagnetic state. Therefore, it becomes a crucial starting point to first unravel the mechanism for the magnetically ordered state.

However, unlike the high- $T_{C}$ cuprates where the antiferromagnetic Mott insulating state can be well interpreted from the strong coupling limit [27], intensive debates persist on whether the magnetic phases can be understood from weak coupling itinerant limit [21,28-31] or from the strong coupling localized limit [32-35] since the discovery of the first high- $T_{C}$ iron-based superconductor LaFeAsO. Due to the similarities between these two high- $T_{C}$ superconductors, such as the phase diagrams in the temperaturedoping plane and the layered lattice structures, a common origin of the high- $T_{C}$ superconductivity as well as the magnetism in both copper-based and iron-based superconductors is highly desirable. This is actually one of the reasons why the theories based on the strong coupling limit are competing with those based on the itinerant limit. Though various theoretical and experimental investigations point to a possible scenario of magnetism that exchange interactions between local spins play essential roles [32,36-40], till now, no direct evidence can confirm the existence of local spins.

On the other hand, remarkable differences are present between these two high- $T_{C}$ superconductors, for example, while the undoped cuprates are antiferromagnetic Mott insulators which can be well described by a one-band Hubbard model, the parent states of iron-based high- $T_{C}$ superconductors are antiferromagnetic metals with multibands crossing the Fermi level. As all the $3 d$ orbitals of Fe ions give contributions to the bands crossing the Fermi level, it is evident that the itinerant electrons should be responsible for the magnetism [21,41-43]. In fact, the itinerant point of view is supported by various theoretical and experimental results [21,28-31,44-48].

Although a compromise between these two limits was proposed that itinerant electrons and localized spins may coexist in the iron-based superconductors [49-56], more unambiguous evidences from experiments besides the angle-resolved photoemission spectroscopy study [57] are required to confirm such a coexistence. Furthermore, the mechanism for the coexistence has to be revealed beyond the slave-spin mean-field calculations [49]. After all, more sophisticate investigations like dynamical mean field theory studies did not support the localization of any electrons in $3 d$ orbitals $[58,59]$.

In this paper, we will first briefly review recent experimental investigations on the antiferromagnetic states in iron-based superconductors. Then, we will focus on a review of theoretical results presented by some of the authors and their collaborators and further supply new findings, which show clear evidences that magnetism in iron-based superconductors is closely related to the itinerant electrons. Our results imply that it is necessary to take into account the itinerant electrons if one would like to properly describe the physics of iron-based superconductors. However, the correlation effect can not be completely ignored as it can strongly suppress the magnetic moment, which may be responsible for the small magnetic moment observed experimentally in iron pnictides.

The remaining paper is organized as follows. In Sec. 2, experiments on the antiferromagnetic phases in different families of iron-based superconductors are presented, including effects of doping, substitution, and applying external pressure. In Sec. 3, the theoretical results based on calculations from first principles and a simplified effective model which can capture the essential physics of ironbased superconductors are shown. Conclusions are given in Sec. 4.

\section{Experimental results}

Up to date, a large number of iron-based superconductors have been discovered. According to the neighboring anions surrounding the irons, these iron-based materials can be cataloged into two groups, namely, iron pnictides (FePn) and iron chalcogenides (FeCh). For FePn, it can be further divided into 4 families based on their chemical formulas, including 111 (like LiFeAs and NaFeAs) [10-12], 1111 (like REOFeAs with RE = rare earth elements and AEFFeAs with $\mathrm{AE}=\mathrm{Ca}$, Sr) [1,4-6,60,61], 122 (like $\mathrm{AEFe}_{2} \mathrm{As}_{2}$ where $\mathrm{AE}=\mathrm{Ca}, \mathrm{Sr}, \mathrm{Ba}$ ) [7-9] and 21311 (like $\mathrm{Sr}_{2} \mathrm{VO}_{3} \mathrm{FeAs}$ ) [13]. For FeCh, there are two different structures, such as $\mathrm{FeSe}_{1-\chi} \mathrm{Te}_{x}$ [14-16], called 11 family, and $\mathrm{A}_{x} \mathrm{Fe}_{2-y} \mathrm{Se}_{2}(\mathrm{~A}=\mathrm{K}, \mathrm{Cs}, \mathrm{Rb}$ and $\mathrm{Tl}$, etc.) [62,63,65-67], named $122 *$ family. All the above families consist of either alternatively stacked $\mathrm{FePn} / \mathrm{FeCh}$ layers and other layers or simply stacked FeCh layers. Unlike the cuprates where 
only Néel ordered antiferromagnetic states are observed, different magnetic patterns have been reported in different families of the iron-based superconductors. Moreover, not only by charge-carrier doping but also by applying external pressure or isoelectronic substitution, the long range antiferromagnetic order can be suppressed and accordingly the superconductivity arises in the iron-based superconductors. Due to the complexity of the magnetic states in the ironbased superconductors, we will first briefly review the experimental results of the widely studied families.

\subsection{FePn}

Typically, collinear antiferromagnetic ordered states are found in most of the Fe-As compounds, except for LiFeAs $[10,11]$ and $\mathrm{KFe}_{2} \mathrm{As}_{2}[64,68]$. Below the spin density wave transition temperature [69], the spins on irons are arranged ferromagnetically along the orthorhombic $b$ axis and antiferromagnetically along the $a$ and $c$ axes (note that $a>b$ here) [70]. All the Fe-P compounds are found to be nonmagnetic, such as LaOFeP [71], LiFeP [72] and $\mathrm{BaFe}_{2} \mathrm{P}_{2}$ [73].

2.1.1. 1111 structure. The magnetic moments of $\mathrm{ReOFeAs}$ detected in experiments are very small. Inelastic neutron scattering measurements indicate an Fe moment of $0.35 \mu_{B}$ [74] for $\operatorname{Re}=\operatorname{Pr}, 0.36 \mu_{B}$ for $\operatorname{Re}=\mathrm{La}$ [75], $0.25 \mu_{B}$ for $\mathrm{Re}=\mathrm{Nd}$ [76], and $0.8 \mu_{B}$ for $\mathrm{Re}=\mathrm{Ce}$ [77]. The magnetic transitions occur around $130 \mathrm{~K}$, preceded by the structure phase transitions from tetragonal to orthorhombic state at about $150 \mathrm{~K}$ [69]. Substitution of oxygen by fluorine, served as electron doping to the Fe-As plane, can suppress both the antiferromagnetic order and structure distortion in ReOFeAs compounds [75,78,79]. By applying external pressure, it is found that the magnetic transitions are suppressed. For example, the undoped LaOFeAs exhibits a magnetic phase transition at $134 \mathrm{~K}$ [80] which decreases at a rate of $-13.7 \mathrm{~K} / \mathrm{GPa}$ as a function of applying pressure [81]. For undoped SmOFeAs, although the magnetic transition also occurs at around $130 \mathrm{~K}$, similar to undoped LaOFeAs, it is found that magnetic transition decreases with pressure at a rate of $-6 \mathrm{~K} / \mathrm{GPa}$ [81], changing more slowly than LaOFeAs.

2.1.2. 122 structure. Inelastic neutron scattering measurements on $\mathrm{AEFe}_{2} \mathrm{As}_{2}(\mathrm{AE}=\mathrm{Ba}, \mathrm{Ca}, \mathrm{Sr})$ parent compounds show larger magnetic moments on $\mathrm{Fe}$ than those in the 1111 family. The magnetic moment is $0.99 \mu_{B}$ in $\mathrm{BaFe}_{2} \mathrm{As}_{2}$ single crystal [82], $0.94 \mu_{B}$ in $\mathrm{SrFe}_{2} \mathrm{As}_{2}$ single crystal [83] and $0.8 \mu_{B}$ in $\mathrm{CaFe}_{2} \mathrm{As}_{2}$ [84] single crystal. Magnetic transition from nonmagnetic state to the collinear antiferromagnetic state and structure transition from $I 4 / \mathrm{mmm}$ tetragonal to Fmmm orthorhombic phase are found to occur at the same temperature in undoped $\mathrm{AEFe}_{2} \mathrm{As}_{2}$ [69]. In various substitution cases such as $\mathrm{BaFe}_{2}\left(\mathrm{As}_{1-\chi} \mathrm{P}_{x}\right)_{2}$ [85], $\mathrm{Ba}_{1-\chi} \mathrm{K}_{x} \mathrm{Fe}_{2} \mathrm{As}_{2} \quad$ [86,87], $\mathrm{Ba}\left(\mathrm{Fe}_{1-\chi} \mathrm{Co}_{x}\right)_{2} \mathrm{As}_{2}$ [88], $\mathrm{Ba}\left(\mathrm{Fe}_{1-\chi} \mathrm{Ru}_{\chi}\right)_{2} \mathrm{As}_{2}$ [89] and $\mathrm{Ba}\left(\mathrm{Fe}_{1-\chi} \mathrm{Ni}_{\chi}\right)_{2} \mathrm{As}_{2}$ [90,91], it is found that the critical temperature of magnetic and struc- tural phase transitions decreases. Separation of these two transitions is found in some critical doping level. In the investigations of applying external pressure, it is shown that the magnetic and structure transitions are suppressed while superconductivity is induced [92]. And it is also proposed from a study of comparisons between pressure and doping effects that structural distortion or change of the Fermi surface nesting, rather than the charge carriers doping, may be responsible for the suppression of magnetic moments and appearance of superconductivity in $\mathrm{BaFe}_{2} \mathrm{As}_{2}$ [93].

2.1.3. 111 structure. LiFeAs shows superconductivity at $18 \mathrm{~K}$ without magnetic and structural transitions [10]. It is found from angle-resolved photoemission spectroscopy study that the Fermi surfaces are poorly nested in LiFeAs [11]. The inelastic neutron scattering and nuclear magnetic resonance studies pointed out that LiFeAs exhibits strong antiferromagnetic fluctuations [94,95]. The magnetic moment of $\mathrm{NaFeAs}$ is the smallest among all the iron-based superconductors which shows long range antiferromagnetic order at low temperature. It is around $(0.09 \pm 0.04) \mu_{B}$ from neutron scattering investigation [96] and is estimated to be $\sim 0.1-0.2 \mu_{B}$ from $\mu$ SR measurement [12]. Stoichiometric $\mathrm{NaFeAs}$ is not a bulk superconductor and can be tuned into superconducting state by small Na deficiency [96-98] or by replacing Fe by either Co or Ni [99].

\section{2. $\mathrm{FeCh}$}

2.2.1. 11 structure. $\mathrm{FeCh}$ are environmentally friendly compared to the FePn compounds. The lattice structure of 11 family is simpler than that of the other families in ironbased superconductors. It consists of stacked $\mathrm{FeCh}$ layers only. Contrast to the Fe-As materials, $\mathrm{Fe}_{1+\chi} \mathrm{Te}$ is found to possess a bicollinear antiferromagnetic state at $x<0.12$ and the magnetically ordered wave vector becomes incommensurate as $x$ becomes larger [100-103]. The angleresolved photoemission spectroscopy experiments did not find the corresponding $(\pi, 0)$ nested Fermi surface topology [104]. The measured magnetic moment of $\mathrm{Fe}$ in $\mathrm{Fe}_{1+\gamma} \mathrm{Te}$ is about $2.25 \mu_{B}$ [103], larger than those of Fe-As compounds. Recently it is found that $\mathrm{Fe}_{1.03} \mathrm{Te}$ becomes ferromagnetic with application of pressure and the magnetic moment per Fe atom reaches about $3 \mu_{B}$ at the highest pressure investigated [105]. $\mathrm{Fe}_{1+\gamma} \mathrm{Se}$ is nonmagnetic and shows tetragonal to orthorhombic structure transition at $90 \mathrm{~K}$ [106]. Superconducting transition temperature $T_{C}$ of $\mathrm{Fe}_{1+\chi} \mathrm{Se}$ gets maximum of $37 \mathrm{~K}$ at $7 \mathrm{GPa}$ with application of hydrostatic pressure [107]. While $\mathrm{Fe}_{1+\chi} \mathrm{Te}$ is not superconducting, the substitution of Te by Se can tune $\mathrm{Fe}_{1.02} \mathrm{Se}_{x} \mathrm{Te}_{1-x}$ compound from $(\pi, 0)$ magnetically ordered state to superconducting state with $(\pi, \pi)$ magnetic resonance in the range of $0<x<0.5$ [108].

2.2.2. $122 *$ structure. Alkali iron selenide superconductors $\mathrm{A}_{x} \mathrm{Fe}_{2-y} \mathrm{Se}_{2}$ with $T_{C}$ around $30 \mathrm{~K}[17,65-67]$ are isostructural to the 122 iron-pnictide $\mathrm{AEFe}_{2} \mathrm{As}_{2} . \mathrm{A}_{x} \mathrm{Fe}_{2-y} \mathrm{Se}_{2}$ 
compounds show insulating or semiconducting properties. $\mathrm{K}_{2} \mathrm{Fe}_{4} \mathrm{Se}_{5}\left(\mathrm{~K}_{0.8} \mathrm{Fe}_{1.6} \mathrm{Se}_{2}\right)$ with $\sqrt{5} \times \sqrt{5} \mathrm{Fe}$ vacancy order has the largest magnetic moment of $3.31 \mu_{B}$ per Fe atom among all the antiferromagnetic ordered iron-based superconductors, and the Néel temperature is up to $560 \mathrm{~K}$ [67]. It is demonstrated that $\mathrm{KFe}_{2} \mathrm{Se}_{2}$ with $\sqrt{2} \times \sqrt{2}$ charge ordering is the parent compound. When interfaced with $\mathrm{K}_{2} \mathrm{Fe}_{4} \mathrm{Se}_{5}, \mathrm{KFe}_{2} \mathrm{Se}_{2}$ becomes superconducting with $\sqrt{2} \times \sqrt{5}$ charge ordering [109].

\section{Theoretical results}

From above review, several questions arise: i) Normally, the iron should be in the high spin state with $S=2$ due to the Hund's rule coupling. So what is the origin of the reduced magnetic moment on each iron, especially in iron pnictides? ii) Contrast to the collinear antiferromagnetic state detected in iron pnictides, $\mathrm{Fe}_{1+\chi} \mathrm{Te}$ exhibits unique bicollinear antiferromagnetic state at low temperature. So is the itinerant scenario still valid if the Fermi surface robustly shows nesting wave vector at $(\pi, \pi)$ ? iii) Various ways such as substitution, applying pressure and charge carrier doping can suppress the antiferromagnetic phase and consequently either induce the superconductivity or become the nonmagnetic state. Can these transitions be accounted for from the itinerant picture? In this section, we will review the theoretical investigations published by some of the authors and their collaborators [29,110-115] which are related to the above questions.

\subsection{Origin of reduced magnetic moment}

The reduced magnetic moment observed experimentally in iron pnictides is usually ascribed to spin frustration. However, the work done by some of the authors and their collaborators [110] proposed a completely new scenario that coupling between weakly and strongly frustrated bands plays an essential role in the reduced magnetic moment. The coexistence of weakly and strongly frustrated bands is in fact a common feature in iron-based superconductors as shown in Table 1 of Ref. 116. In order to prove the validity of this scenario, a two-band Hubbard model was used which is defined as follows,

$$
\begin{aligned}
H= & -\sum_{\langle i j\rangle,\langle\langle i j\rangle\rangle, \gamma \sigma} t_{i j, \gamma} c_{i \gamma \sigma}^{\dagger} c_{j \gamma \sigma}+U \sum_{i \gamma} n_{i \gamma \uparrow} n_{i \gamma \downarrow}+ \\
& +\left(U^{\prime}-\frac{J}{2}\right) \sum_{i \gamma>\gamma^{\prime}} n_{i \gamma} n_{i \gamma^{\prime}}-2 J \sum_{i \gamma>\gamma^{\prime}} S_{i \gamma}^{Z} S_{i \gamma^{\prime}}^{Z}
\end{aligned}
$$

where $t_{i j, \gamma}=t_{\gamma}\left(t_{\gamma}^{\prime}\right)$ is the intraorbital hopping integral between nearest-neighbor (next-nearest-neighbor) sites denoted by $\langle i j\rangle(\langle\langle i j\rangle\rangle)$ with orbital indices $\gamma=\alpha, \beta$ in units of $t$. $U, U^{\prime}$ and $J$ are the intraband, interband Coulomb interaction and Hund's coupling, respectively, which fulfill the rotational invariance condition $U=U^{\prime}+2 J$. Here $J / U=0.25$ was chosen. The interband hybridizations, pair-hopping and spin flip terms are ignored for simplicity. $c_{i \gamma \sigma}^{\dagger}\left(c_{i \gamma \sigma}\right)$ creates (annihilates) an electron in orbital $\gamma$ of site $i$ with spin $\sigma . n_{i \gamma \sigma}$ is the occupation operator, while $n_{i \gamma}=n_{i \gamma \uparrow}+n_{i \gamma \downarrow}$, and $S_{i \gamma}^{Z}=\left(n_{i \gamma \uparrow}-n_{i \gamma \downarrow}\right) / 2$ the spin operator of $z$ direction.

The model was solved by the two-sublattice dynamical mean-field theory method which includes the local quantum fluctuation effects and can account for antiferromagnetic state. The weak coupling continuous time quantum Monte Carlo method [117,118] is employed as an impurity solver. The Bethe lattice with infinite coordination is used and the self consistent conditions are given by

$$
\begin{aligned}
& G_{0, A, \sigma}^{-1}=i \omega_{n}+\mu-t_{\gamma}^{2} G_{B, \sigma}-t_{\gamma}^{\prime 2} G_{A, \sigma}, \\
& G_{0, B, \sigma}^{-1}=i \omega_{n}+\mu-t_{\gamma}^{2} G_{A, \sigma}-t_{\gamma}^{\prime 2} G_{B, \sigma},
\end{aligned}
$$

where $G_{0}^{-1}$ and $G$ are the Weiss fields and the local Green's functions, respectively. Here A and B label two different sublattices with opposite spins, $\omega_{n}$ is the Matsubara frequency, and $\mu$ is the chemical potential which controls the filling.

In Fig. 1, the results for the staggered magnetization per band are presented as a function of $U / t$ for $T / t=1 / 16$
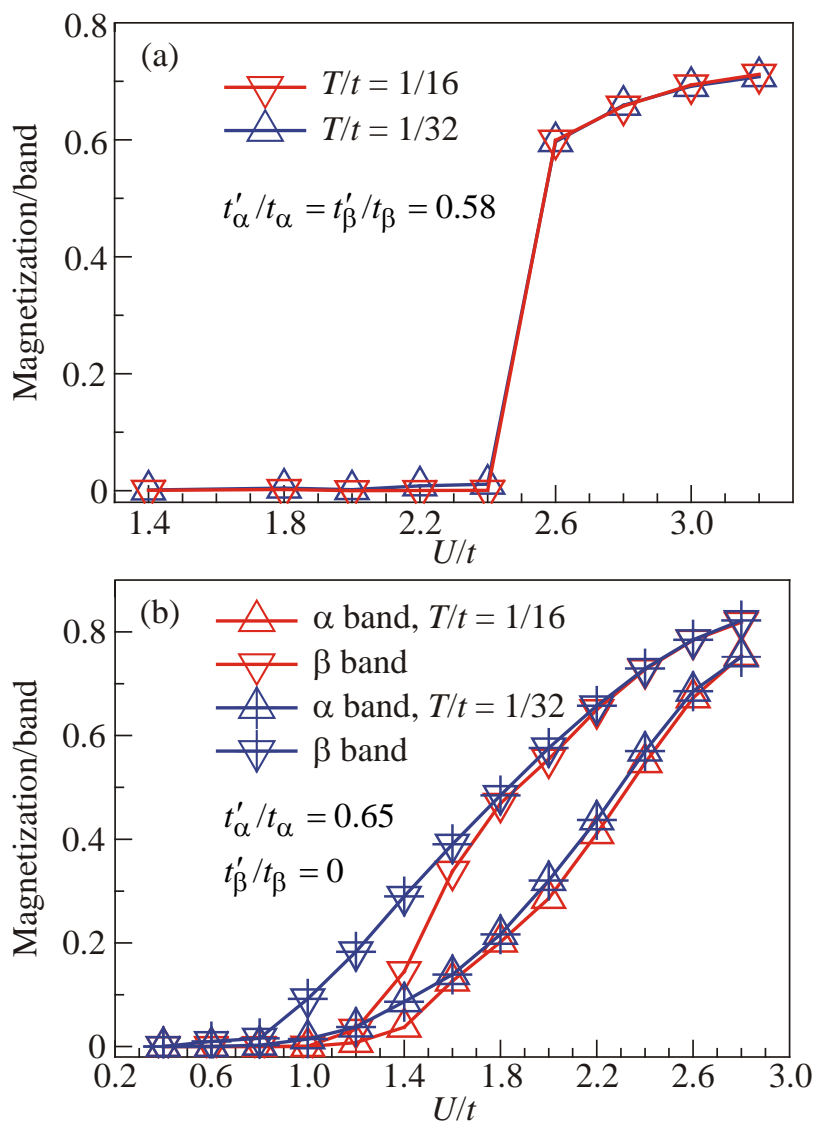

Fig. 1. (Color online) Magnetization per band of a two-band Hubbard model at two temperatures calculated by dynamical mean-field theory with weak coupling continuous time quantum Monte Carlo as a function of interaction strength $U$ with (a) two bands equally frustrated and (b) one band frustrated, the other unfrustrated. From Ref. 110. 
and $1 / 32$. In order to find out which is the mechanism for the reduced magnetic moment, two cases were studied. One is the case of pure spin frustration as shown in Fig. 1(a). In this case, both bands are set to be equally frustrated with the ratio of $t_{\gamma}^{\prime} / t_{\gamma}=0.58$ for $\gamma=\alpha, \beta$. The other is the case of coupling between frustrated and unfrustrated bands with $t_{\alpha}^{\prime} / t_{\alpha}=0.65$ and $t_{\beta}^{\prime} / t_{\beta}=0.0$, which is shown in Fig. 1(b). Please note that the strength of the frustration is determined by the ratio of $t_{\gamma}^{\prime} / t_{\gamma}$. Below $U / t=2.4$ in Fig. 1(a), the staggered magnetization for both temperatures is negligibly small, indicating a paramagnetic state. As the interaction $U / t$ is increased, for both temperatures a jump is detected around the critical value of $U_{c} / t=2.4$ and the system directly evolves into an antiferromagnetic state with large magnetic moment, indicating that spin frustration alone cannot account for the state with small magnetic moment. However, in Fig. 1(b), a smooth increase is observed in the magnetization with increasing $U / t$ for both bands and for both temperatures. While the magnetization is stronger in unfrustrated band than that in frustrated band, the magnetic moment in both bands remains small in the intermediate region of $U / t$, which strongly supports the mechanism of coupling between weakly and strongly frustrated bands. Certainly, local quantum fluctuations should also play important roles in stabilizing the antiferromagnetic state with small magnetic moment, as we learn from the comparisons between Hartree-Fock calculations and dynamical mean-field theory calculations [119].

\subsection{Origin of bicollinear antiferromagnetism in $\mathrm{Fe}_{1+\chi} \mathrm{Te}$}

The magnetic state of $\mathrm{Fe}_{1+\chi} \mathrm{Te}$ observed from neutron diffraction analysis is bicollinear antiferromagnetic, which is completely different from that of iron pnictides. It requires a magnetic instability at wave vector of $(\pi, 0)$ if itinerant picture of magnetism is still valid. However, the hole and electron Fermi surfaces observed from angleresolved photoemission spectroscopy study are still located at the center and the corner of the Brillouin zone, respectively, and separated by a wave vector of $(\pi, \pi)$ as usually detected in iron pnictides. Such a discrepancy casts doubt on the applicability of itinerant scenario of magnetism to the 11 family and further leads to a question whether one can establish a common theory for the magnetism and superconductivity in iron pnictides and iron chalcognides.

Recently, it was suggested by the authors [111] that magnetism in $\mathrm{Fe}_{1+\chi} \mathrm{Te}$ still has its itinerant origin even without Fermi surface nesting, provided orbital modulation of particle-hole excitations as well as the excess Fe which donates extra electrons are considered. Magnetic exchange coupling between excess Fe and in-plane Fe further stabilizes the bicollinear antiferromagnetic order.

The scenario is verified by investigating the Pauli susceptibility, which is defined as [120]

$$
\begin{aligned}
\chi_{s t}^{u w}(q, \omega)= & -\frac{1}{N} \sum_{k, \mu v} \frac{a_{\mu}^{s}(k) a_{\mu}^{u *}(k) a_{v}^{w}(k+q) a_{v}^{t *}(k+q)}{\omega+E_{v}(k+q)-E_{\mu}(k)+i 0^{+}} \times \\
& \times\left[f\left(E_{v}(k+q)\right)-f\left(E_{\mu}(k)\right)\right]
\end{aligned}
$$

where $\mu$ and $v$ are the band indices and $q$ and $k$ are the momentum vectors in the Brillouin zone; $u, w, s, t$ are the orbital indices varying from 1 to 5 which represent five $3 d$ orbitals of Fe (orbital 1: $d_{x y}, 2: d_{y z}, 3: d_{x z}, 4: d_{x^{2}-y^{2}}, 5: d_{z^{2}}$, where $x, y, z$ refer to those for the original unit cell). $f(E)$ is the Fermi distribution function. The matrix element $a_{\mu}^{s}(k)=\langle s \mid \mu k\rangle$ represents the projection of the $\mu$-th Bloch band onto the $s$-th Wannier orbital at the momentum vector $k$, which is the component of the eigenvectors resulting from the diagonalization of an effective tight-binding Hamiltonian with ten $3 d$ orbitals from two Fe ions and six $5 p$ orbitals from two Te ions. The model parameters of the effective Hamiltonian are derived from the first principles calculations through construction of the maximally localized Wannier orbital basis [121,122]. The full potential linearized augmented plane wave method as implemented in Wien2k [123] is employed to calculate the band structures. The results, independent of exchange-correlation functional one chooses, are obtained within local density approximation. A three-dimensional grid of $128 \times 128 \times 128 \mathrm{k}$ and $q$ points is used in the whole Brillouin zone with a temperature smearing of $0.01 \mathrm{eV}$ for calculating the Pauli susceptibility. The experimental lattice structure [103] is used except when the substitution effect of Se by Te is studied in the system.

It was proposed that the excess Fe which can not be eliminated in the experiments [100-104] plays two roles. One is to provide extra electrons into the Fe-Te layers, which can be viewed as an electron doping. In order to take into account the doping effect, rigid band shift is used in the investigations. Figure 2 shows momentum dependent Pauli susceptibility of $d_{x z}$ and $d_{x^{2}-y^{2}}$ orbitals along the path of $(0,0, \pi) \rightarrow(\pi, 0, \pi) \rightarrow(\pi, \pi, \pi) \rightarrow(0,0, \pi)$ at different Fermi energy shifts, corresponding to different number of excess Fe varying from $x=0$ to $x=0.2$. Here it is reasonably assumed that each excess Fe contributes 2 extra electrons into the Fe-Te layers. Without shift or as the shifts are small, like $\Delta E_{F}=0$ and 0.045 , both $\chi_{33}^{33}$ and $\chi_{44}^{44}$ show dominant peaks around $(\pi, \pi, \pi)$. While the peak in $\chi_{44}^{44}$ is slight enhanced at $\Delta E_{F}=0.045$, compared to the case of $\Delta E_{F}=0$, that in $\chi_{33}^{33}$ is strongly suppressed. At $\Delta E_{F}=0.065,0.075,0.085$, well-defined peak centered at $(\pi, 0, \pi)$ appears in $\chi_{33}^{33}$, indicating that bicollinear antiferromagnetic state has its itinerant origin. The peak at $(\pi, \pi, \pi)$ in $\chi_{44}^{44}$, however, remains almost unchanged at these $\Delta E_{F}$. Further increasing $\Delta E_{F}$ to $0.105,0.12,0.15$, the peak around $(\pi, \pi, \pi)$ in $\chi_{44}^{44}$ rapidly decreases, while the peak previously right at $(\pi, 0, \pi)$ in $\chi_{33}^{33}$ moves to an incommensurate wave vector, which is consistent with experimental finding of transitions from bicollinear antiferromagnetic states to 

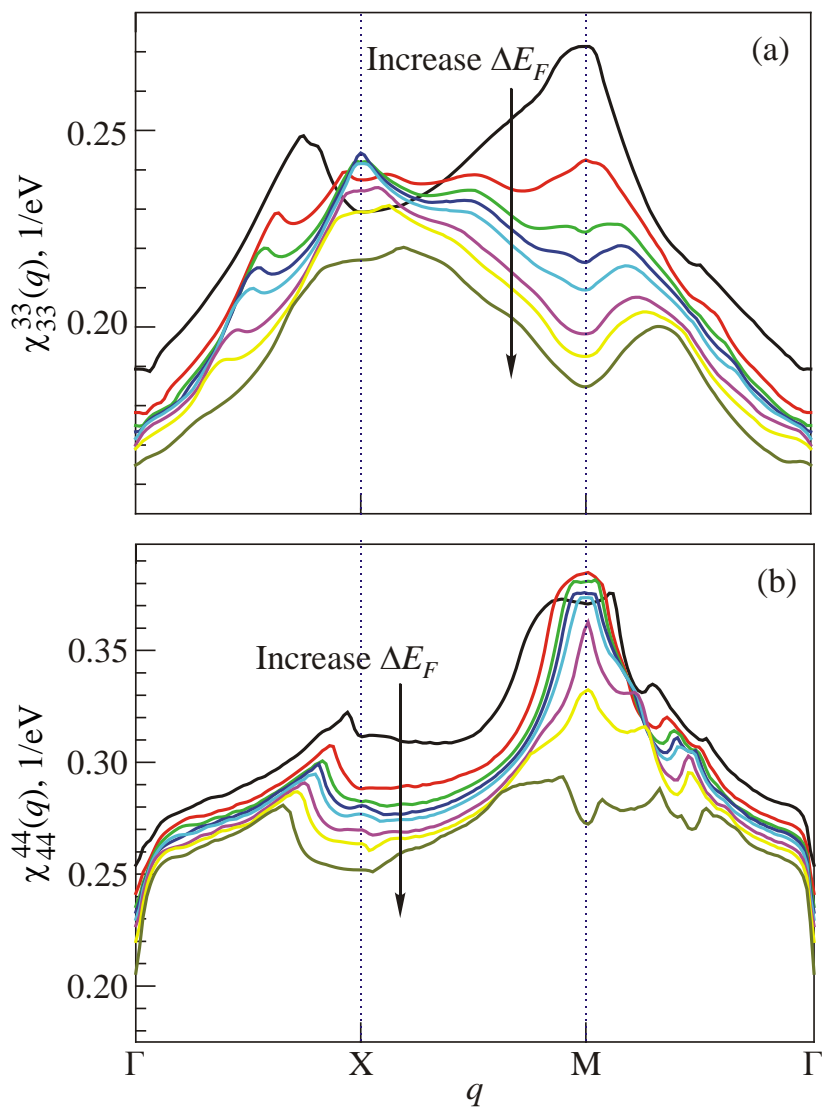

Fig. 2. (Color online) Evolution of particle-hole excitations in Pauli susceptibility as a function of shifted Fermi energies. (a) $\chi_{33}^{33}(q, \omega=0)$ with 3 representing $d_{x z}$ orbital. (b) $\chi_{44}^{44}(q, \omega=0)$ with 4 denoting $d_{x^{2}-y^{2}}$ orbital. $\Delta E_{F}=0,0.045,0.065,0.075$, $0.085,0.105,0.12,0.15 \mathrm{eV}$, corresponding to the $\mathrm{Fe}_{1+{ }_{\chi}} \mathrm{Te}$ compounds with $x=0,0.06,0.09,0.10,0.12,0.14,0.16,0.20$. From Ref. 111.

incommensurate phases as number of excess Fe increases [100-103]. The larger the shift is, the farther the peak away from $(\pi, 0, \pi)$ which is also consistent with experiments. The susceptibilities of $d_{z}$ and $d_{x y}$ orbitals are negligibly small compared to those of $d_{x z}$ and $d_{x^{2}-y^{2}}$ orbitals and that of $d_{y z}$ orbital shows a mirror symmetry to $\chi_{33}^{33}(q, \omega=0)$ with respect to $q_{x}=q_{y}$. Therefore, these three susceptibilities are not shown here.

Although it was shown that the bicollinear antiferromagnetic state in $\mathrm{Fe}_{1+\chi}$ Te has its itinerant origin, one may still wonder why the weaker instability of $d_{x z}$ orbital at $(\pi, 0, \pi)$ wins the competition over the stronger instability of $d_{x^{2}-y^{2}}$ orbital at $(\pi, \pi, \pi)$. This is in fact due to the second role of the excess $\mathrm{Fe}$. In addition to contributing extra electrons, the excess $\mathrm{Fe}$ also provides a magnetic ion which is strongly coupled with the in-plane Fe. Due to this magnetic interaction, the bicollinear antiferromagnetic state is further stabilized, rather than the collinear antiferromagnetic state. This explanation was justified by spin polarized density functional theory calculations on a supercell with 16 in-plane Fe and 1 interstitial Fe. Without
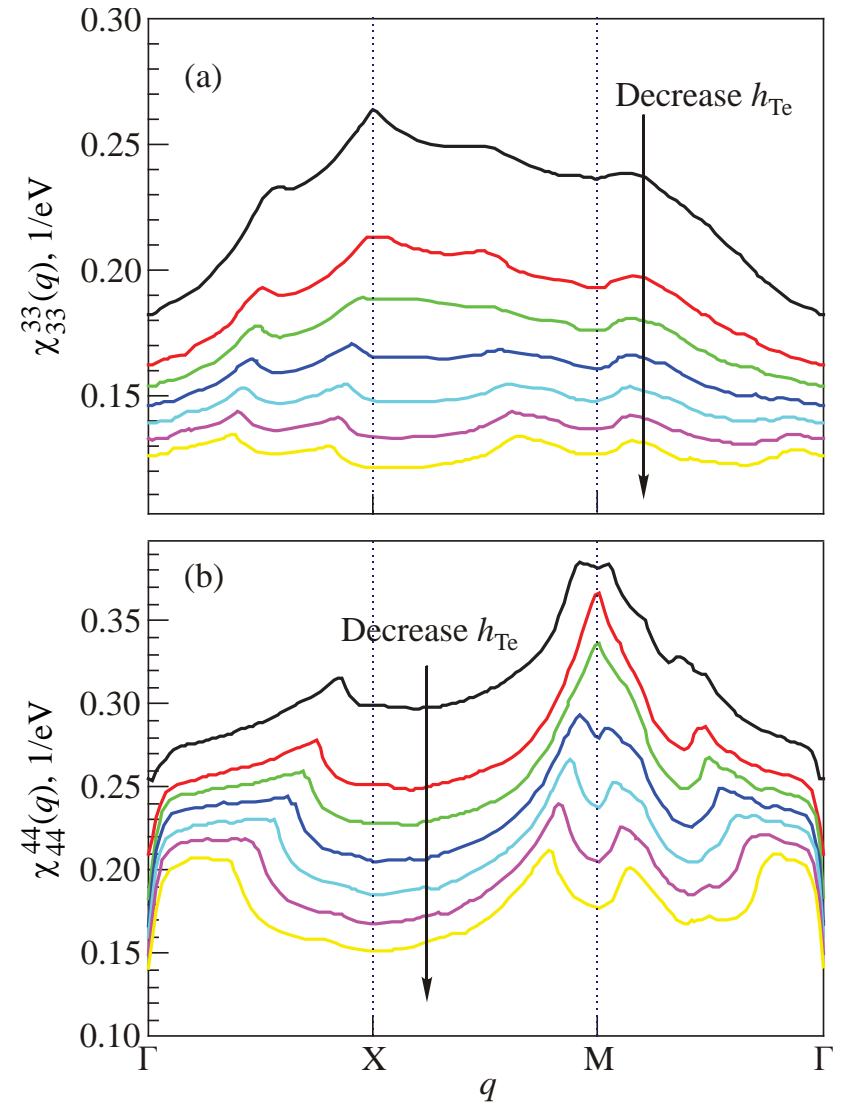

Fig. 3. (Color online) Evolution of particle-hole excitations in Pauli susceptibility as a function of Te-height measured from Fe plane. (a) $\chi_{33}^{33}(q, \omega=0)$ with 3 representing $d_{x y}$ orbital. (b) $\chi_{44}^{44}(q, \omega=0)$ with 4 denoting $d_{x^{2}-y^{2}}$ orbital. Te-height from $h_{\mathrm{Te}}=1.8 \AA$ to $1.52 \AA$ in the interval of $0.04 \AA$. From Ref. 111.

excess $\mathrm{Fe}$, the magnetic ground state of $\mathrm{Fe}_{16} \mathrm{Te}_{16}$ is of collinear antiferromagnetic order within local density approximation, which is inconsistent with the experiments. After putting one excess Fe into the interstitial of the supercell with the position according to the neutron diffraction experiments, the bicollinear antiferromagnetic state turns to be the ground state, indicating that the bicollinear antiferromagnetic state is stabilized by excess magnetic Fe. However, if the magnetic moment is tuned off, which can be realized by changing the interstitial magnetic Fe to nonmagnetic $\mathrm{Zn}$, the collinear antiferromagnetic state becomes the ground state within local density approximation. This is a clear evidence that existence of interstitial ion with magnetic moment is crucial for stabilizing the bicollinear antiferromagnetic state.

Furthermore, evolution of the particle-hole excitations in the Pauli susceptibility as a function of Te-height measured from the Fe plane was also investigated. This can be effectively viewed as the substitution of Te by Se since Seheight is much lower than Te-height. The number of extra electrons is kept to be $0.2 / \mathrm{Fe}$. From Fig. 3 one can see that 
lowering Te-height rapidly suppresses the $(\pi, 0, \pi) /(0, \pi, \pi)$ instability, this may be the reason why bicollinear magnetic order disappears as substitution of Te by Se in experiment [108]. Meanwhile the $(\pi, \pi, \pi)$ instability in $d_{x^{2}-y^{2}}$ orbital remaining relatively large may be the source of superconductivity with $(\pi, \pi)$ magnetic resonance observed in experiment [108]. These results may indicate that different orbitals are responsible for different electronic properties, like magnetism or superconductivity in 11 family.

\subsection{Origin of magnetic phase transitions in 122 compounds}

Unlike the cuprates, various ways, like applying external pressure or charge carrier doping, etc., can be used to suppress the magnetic phase and on the other hand enhance possibly the superconducting state. In this part, we will review two typical cases which show strong evidences that the magnetic phase transitions are closely related to itinerant electrons. One is the pressure induced phase transitions occuring in $\mathrm{AEFe}_{2} \mathrm{As}_{2}$ where $\mathrm{AE}=\mathrm{Ca}, \mathrm{Sr}$, and $\mathrm{Ba}$. In order to capture the lattice structures under various pressures, the Car-Parrinello projector-augmented wave molecular dynamics method at constant pressure was employed [124-126]. The details can be found in Ref. 112. Here only the results for $\mathrm{CaFe}_{2} \mathrm{As}_{2}$ are shown. In Figs. 4(a) and (b), the calculated changes of the volume and magnetization of $\mathrm{CaFe}_{2} \mathrm{As}_{2}$ as a function of pressure are presented. The volume decreases gradually with increasing pressure and shows a discontinuous shrinkage at the critical pressure, where the system undergoes a structural phase transition from orthorhombic symmetry to a volume "collapsed" tetragonal symmetry. Meanwhile, the magnetization sharply goes to zero. Such a sudden jump can also be detected in the calculated Pauli susceptibility $\chi_{0}(q, 0)$ at $q=(\pi, \pi, 0)$ as shown Fig. 4(c). Here the $\chi_{0}(q, 0)$ was obtained within the constant matrix element approximation where all the $a_{\mu}^{s}(k)$ are set to be 1 in Eq. (4). The abrupt reduction appearing in the $\chi_{0}(q, 0)$ at the same critical pressure indicates that the structural and magnetic phase transitions in $\mathrm{CaFe}_{2} \mathrm{As}_{2}$ may be ascribed to the suppression of the $(\pi, \pi)$ instability which shows tendency towards collinear antiferromagnetic order.

The second is the substitution of Fe by $\mathrm{Co}$ in $\mathrm{BaFe}_{2} \mathrm{As}_{2}$ [127]. With increase of Co concentration (x), the ground state of $\mathrm{BaFe}_{2-x} \mathrm{Co}_{x} \mathrm{As}_{2}$ evolves from a collinear antiferromagnetic state $(x=0)$ to a paramagnetic state $(x=0.5)$ through an intermediate superconducting state $(x=0.2)$. Via a calculation of Pauli susceptibility $\chi_{0}(q, 0)$, it is also suggested that these phase transitions are closely related to the instability of the itinerant electrons. As is shown in Fig. 5 that at $x=0$ a strong instability in the Pauli susceptibility is present at $q=(\pi, \pi, 0)$ (solid curve), supporting the collinear antiferromagnetic state. At $x=0.2$, a strong suppression of the $q=(\pi, \pi, 0)$ instability (dashed curve) indicates a suppression of the magnetization. However, the

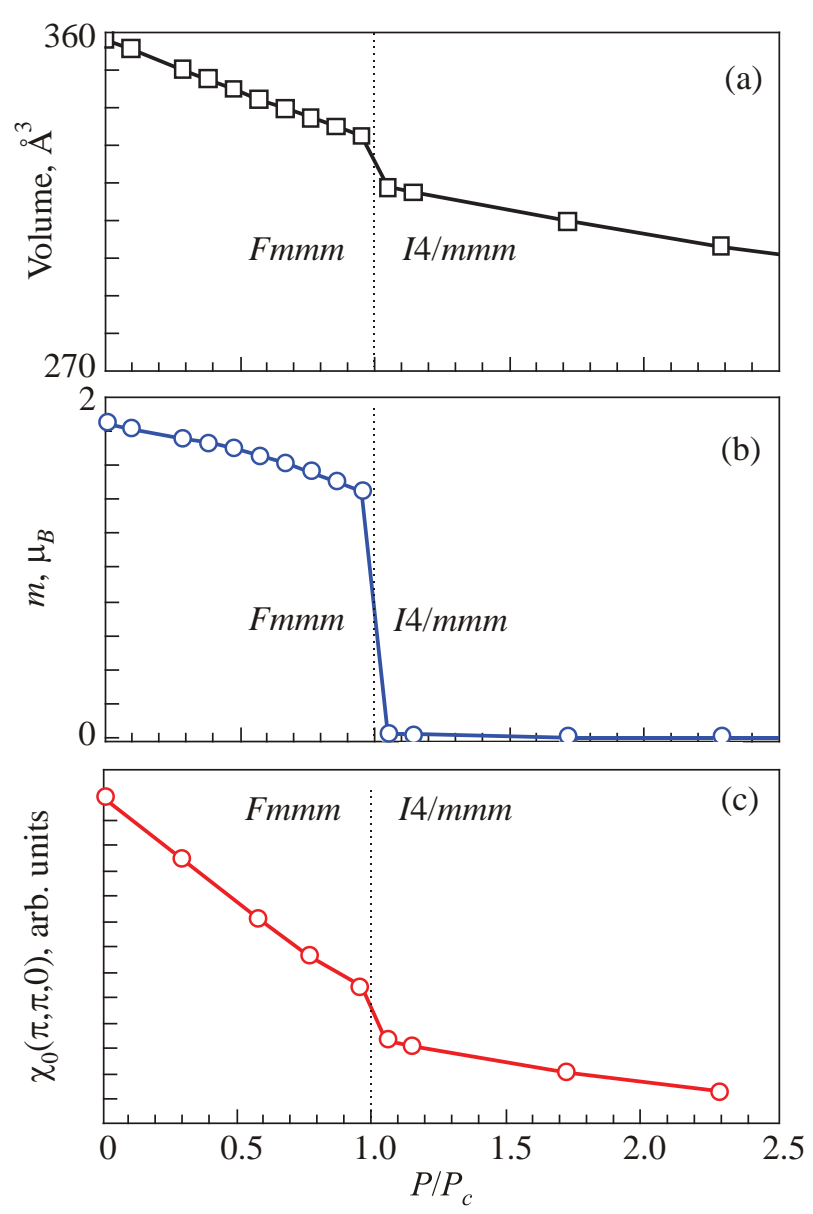

Fig. 4. (Color online) Calculated changes of volume (a), magnetization (b), and Pauli susceptibility $\chi_{0}(q)$ at $q=(\pi, \pi, 0)$ within the constant matrix element approximation (c) as a function of applied external pressure normalized to the critical pressure for $\mathrm{CaFe}_{2} \mathrm{As}_{2}$. The phase boundary between Fmmm and I4/mmm is indicated by the vertical dashed line. From Ref. 112.

superconducting state which, according to the spin fluctuation theory [128], is related to the instabilities around $q=(\pi, \pi, 0)$ may in such a situation be more favorable than

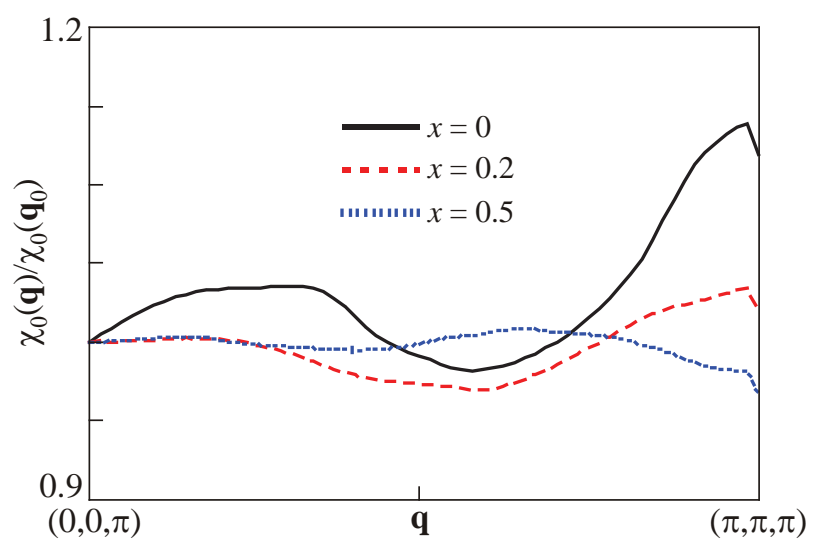

Fig. 5. (Color online) Normalized q-dependent Pauli susceptibilities of $\mathrm{BaFe}_{2-x} \mathrm{Co}_{x} \mathrm{As}_{2}$ at fixed $q_{z}=\pi$ along the [110] direction calculated within generalized gradient approximation. Here $x=0,0.2,0.5$. The normalization factors are the susceptibilities of each compound at $\mathbf{q}_{0}=(0,0, \pi)$. From Ref. 113 . 
magnetization. At $x=0.5$, the Pauli susceptibility $\chi_{0}(q, 0)$ becomes featureless and no obvious instability is present (dotted curve), leading to the disappearance of both magnetic ordering and superconductivity.

\subsection{Origin of collinear antiferromagnetic states in 111 and 1111 families}

In 111 family, it was found from experiments that, while NaFeAs shows collinear antiferromagnetic order at low temperature [10,96,99], it becomes nonmagnetic as $\mathrm{Na}$ is replaced by Li. Similar situation appears in 1111 family. While LaOFeAs exhibits collinear antiferromagnetic order, the replacement of As by $\mathrm{P}$ leads to a nonmagnetic state. In Ref. 29, an itinerant scenario was provided to understand the difference in the magnetic states of 1111 families. It is ascribed to a reduction of instability at $q=(\pi, \pi)$ and an increasing competition between the instabilities at $q=(\pi, \pi)$ and $q=(0,0)$ in LaOFeP, compared to those in LaOFeAs. Here we will show that the phenomena appearing in 111 family can be also understood from the itinerant limit.

We calculate orbitally resolved Pauli susceptibilities of LiFeAs and NaFeAs according to Eq. (4). The results are presented in Fig. 6. Again we find that Pauli susceptibilities from $d_{x y}$ and $d_{z} 2$ orbitals are negligibly small com-
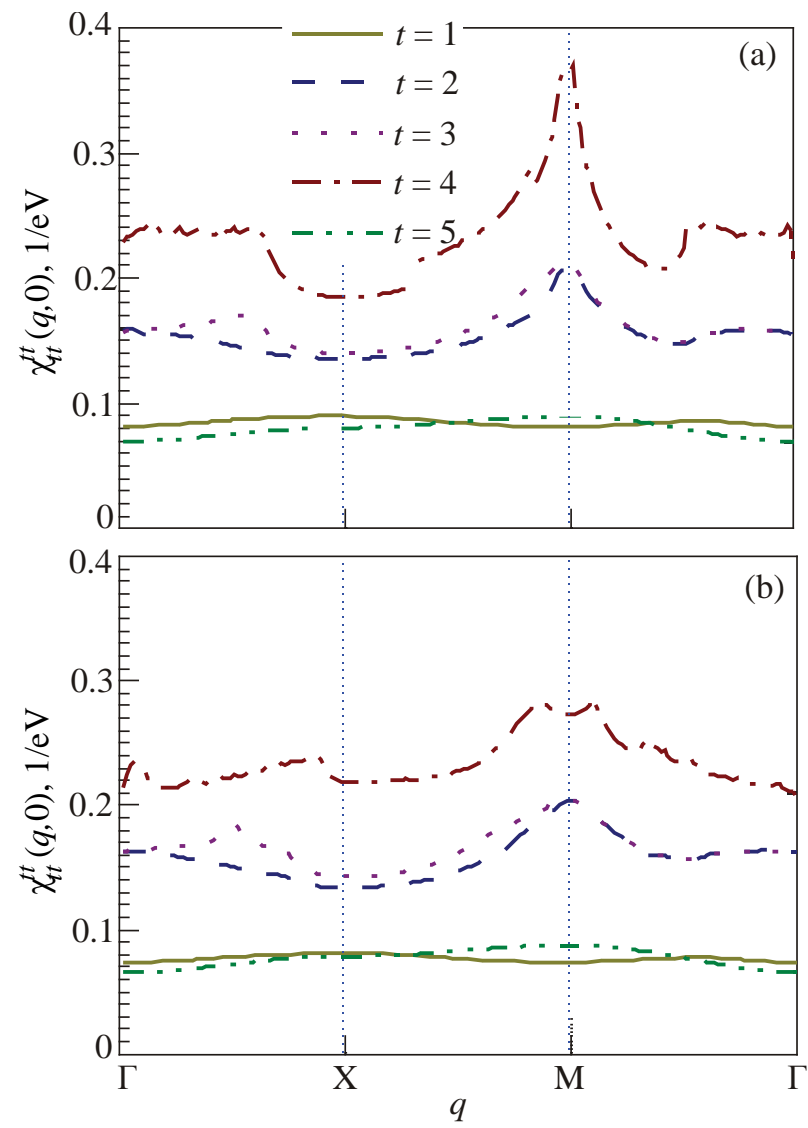

Fig. 6. (Color online) Pauli susceptibilities of NaFeAs (a) and LiFeAs (b) for all five Fe $3 d$ orbitals. Here 1, 2, 3, 4, 5 represents $d_{x y}, d_{y z}, d_{x z}, d_{x^{2}-y^{2}}, d_{z^{2}}$ orbitals, respectively, where $x, y, z$ refer to those for the original unit cell. pared to those from $d_{x z} / d_{y z}$ and $d_{x^{2}-y^{2}}$ orbitals. The susceptibilities from $d_{x z} / d_{y z}$ are almost the same in both LiFeAs and NaFeAs. However, the susceptibility from $d_{x^{2}-y^{2}}$ orbital shows remarkable difference between these two compounds. A strong peak at $q=(\pi, \pi)$ can be seen in $\mathrm{NaFeAs}$, while it is absent in LiFeAs, strongly indicating that the magnetic properties of these two systems may be controlled by the $d_{x^{2}-y^{2}}$ orbital. Combining the results from 11 systems where $d_{y z} / d_{x z}$ orbitals play important roles in the formation of magnetism and $d_{x^{2}-y^{2}}$ orbital may be responsible for the superconductivity, we propose that orbital differentiation is very important in understanding the physical properties of iron-based superconductors.

Then we will try to understand the effect of different alkali metals and the effect of changing the lattice structure. We use the experimental structures of LiFeAs [10] and $\mathrm{NaFeAs}$ [96]. Then we replace Li by $\mathrm{Na}$ in LiFeAs and replace $\mathrm{Na}$ by $\mathrm{Li}$ in NaFeAs, respectively. From Fig. 7(a) one can see that Pauli susceptibility from $d_{x^{2}-y^{2}}$ orbital becomes significantly smaller when $\mathrm{Na}$ is replaced by $\mathrm{Li}$, implying the importance of the alkali metal to the magnetic properties. From Fig. 7(b), the Pauli susceptibility from $d_{x^{2}-y^{2}}$ orbital does not change much at the wave vector of $(\pi, \pi)$ when $\mathrm{Li}$ is substituted by $\mathrm{Na}$ with experimental
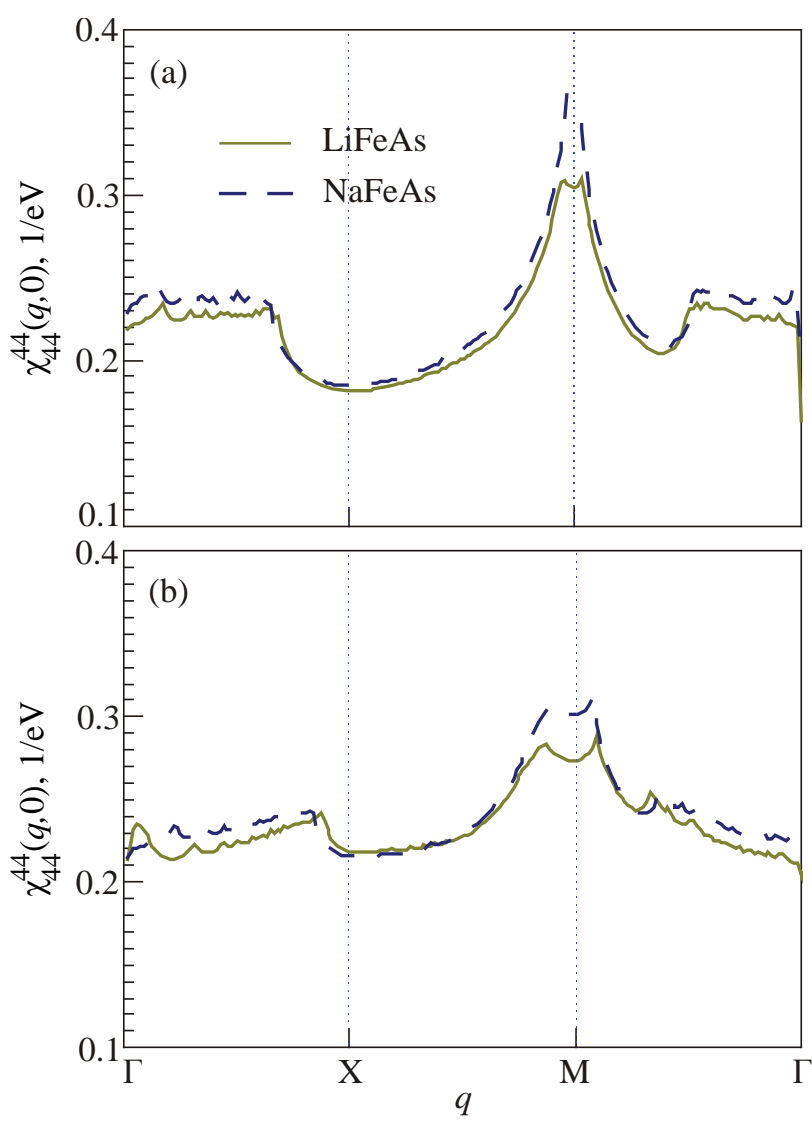

Fig. 7. (Color online) Effects of replacement of $\mathrm{Na}$ by $\mathrm{Li}$ in NaFeAs (a) and Li by Na in LiFeAs (b) on the Pauli susceptibility of $d_{x^{2}-y^{2}}$ orbital. Experimental structures of NaFeAs and LiFeAs are used in (a) and (b), respectively. 
structure of LiFeAs, indicating that the lattice structure also plays an important role in determining the magnetic properties in 111 family.

\section{Conclusions}

In this paper, experimental progresses in the study of antiferromagnetism in iron-based superconductors are briefly reviewed. The theories from the itinerant limit established by the authors and their collaborators are also reviewed, which cover various families of iron-based superconductors, such as 122, 11, 111 and 1111. From above discussions, we can conclude that magnetism in iron-based superconductors has its itinerant origin. The inclusion of orbital degrees of freedom and the differentiation in the orbitals are very important. Strong correlations can further stabilize the small magnetic moment states. However, we do not touch the theory for the magnetism in $122 *$ family, since many uncertainties remain from experimental sides. Most probably, the peculiar magnetic patterns observed experimentally are strongly influenced by the vacancy of the Fe. The theories presented in this paper do not rule out the possibility of existence of local spins. However, up to now, it is still lack of theory for the origin of localized electrons. Dynamical mean-field theory calculations only show heavier effective mass of quasiparticles in all the $3 d$ orbitals as suitable correlation is tuned on, which casts doubt on the scenario of local spins. As dynamical mean field theory can only take into account the local fluctuations, future work should be focused on the multi-orbital physics with inclusion of spatial correlations if one would like to reveal unambiguously the origin of magnetism in iron-based superconductors.

\section{Acknowledgments}

Y.Z. is supported by National Natural Science Foundation of China (No. 11174219), Shanghai Pujiang Program (No. 11PJ1409900), Research Fund for the Doctoral Program of Higher Education of China (No. 20110072110044) and the Program for Professor of Special Appointment (Eastern Scholar) at Shanghai Institutions of Higher Learning as well as the Scientific Research Foundation for the Returned Overseas Chinese Scholars, State Education Ministry.

1. Y. Kamihara, T. Watanabe, M. Hirano, and H. Hosono, J. Am. Chem. Soc. 130, 3296 (2008).

2. Z.A. Ren, W. Lu, J. Yang, W. Yi, X.L. Shen, Z.C. Li, G.C. Che, X.L. Dong, L.L. Sun, F. Zhou, and Z.X. Zhao, Chin. Phys. Lett. 25, 2215 (2008).

3. Q.Y. Wang, Z. Li, W.H. Zhang, Z.C. Zhang, J.S. Zhang, W. Li, H. Ding, Y.B. Ou, P. Deng, K. Chang, J. Wen, C.L. Song, K. He, J.F. Jia, S.H. Ji, Y.Y. Wang, L.L. Wang, X. Chen, X.C. Ma, and Q.K. Xue, Chin. Phys. Lett. 29, 037402 (2012).
4. C. Wang, L.J. Li, S. Chi, Z.W. Zhu, Z. Ren, Y.K. Li, Y.T. Wang, X. Lin, Y.K. Luo, S. Jiang, X.F. Xu, G.H. Cao, Z.A. Xu, Europhys. Lett. 83, 67006 (2008).

5. X.H. Chen, T. Wu, G. Wu, R.H. Liu, H. Chen, and D.F. Fang, Nature 453, 761 (2008).

6. G.F. Chen, Z. Li, D. Wu, G. Li, W.Z. Hu, J. Dong, P. Zheng, J.L. Luo, and N.L. Wang, Phys. Rev. Lett. 100, 247002 (2008).

7. A.S. Sefat, R. Jin, M.A. McGuire, B.C. Sales, D.J. Singh, and D. Mandrus, Phys. Rev. Lett. 101, 117004 (2008).

8. M.S. Torikachvili, S.L. Bud'ko, N. Ni, and P.C. Canfield, Phys. Rev. Lett. 101, 057006 (2008).

9. K. Sasmal, B. Lv, B. Lorenz, A.M. Guloy, F. Chen, Y.Y. Xue, and C.W. Chu, Phys. Rev. Lett. 101, 107007 (2008).

10. J.H. Tapp, Z.J. Tang, B. Lv, K. Sasmal, B. Lorenz, P.C.W. Chu, and A.M. Guloy, Phys. Rev. B 78, 060505 (2008).

11. S.V. Borisenko, V.B. Zabolotnyy, D.V. Evtushinsky, T.K. Kim. Guloy, I.V. Morozov, A.N. Yaresko, A.A. Kordyuk, G. Behr, A. Vasiliev, R. Follath, and B. Büchner, Phys. Rev. Lett. 105, 067002 (2010).

12. D.R. Parker, M.J. Pitcher, P.J. Baker, I. Franke, T. Lancaster, S.J. Blundell, and S.J. Clarke, Chem. Commun. 16, 2189 (2009).

13. X.Y. Zhu, F. Han, G. Mu, P. Cheng, B. Shen, B. Zeng, and H.H. Wen, Phys. Rev. B 79, 220512 (2009).

14. F.C. Hsu, J.Y. Luo, K.W. Yeh, T.K. Chen, T.W. Huang, P.M. Wu, Y.C. Lee, Y.L. Huang, Y.Y. Chu, D.C. Yan, and M.K. Wu, PNAS 105, 38 (2008).

15. Y. Mizuguchi, F. Tomioka, S. Tsuda, T. Yamaguchi, and Y. Takano, Appl. Phys. Lett. 93, 152505 (2008).

16. K.W. Yeh, T.W. Huang, Y.L. Huang, T.K. Chen, F.C. Hsu, P.M. Wu, Y.C. Lee, Y.Y. Chu, C.L. Chen, J.Y. Luo, D.C. Yan, and M.K. Wu, Europhys. Lett. 84, 37002 (2008).

17. J.G. Guo, S.F. Jin, G. Wang, S.C. Wang, K.X. Zhu, T.T. Zhou, M. He, and X.L. Chen, Phys. Rev. B 82, 180520(R) (2010).

18. L.L. Sun, X.J. Chen, J. Guo, P.W. Gao, Q.Z. Huang, H.D. Wang, M.H. Fang, X.L. Chen, G.F. Chen, Q. Wu, C. Zhang, D.C. Gu, X.L. Dong, L. Wang, K. Yang, A.G. Li, X. Dai, H.K. Mao, and Z.X. Zhao, Nature 483, 67 (2012).

19. F. Wang and D.H. Lee, Science 332, 200 (2011).

20. I.I. Mazin, Nature 464, 183 (2010).

21. I.I. Mazin, D.J. Singh, M.D. Johannes, and M.H. Du, Phys. Rev. Lett. 101, 057003 (2008).

22. F. Wang, H. Zhai, Y. Ran, A. Vishwanath, and D.H. Lee, Phys. Rev. Lett. 102, 047005 (2009).

23. R. Thomale, C. Platt, W. Hanke, and B.A. Bernevig, Phys. Rev. Lett. 106, 187003 (2011).

24. X. Lu, C. Fang, W.F. Tsai, Y. Jiang, and J. Hu, Phys. Rev. B 85, 054505 (2012).

25. D.J. Scalapino, Rev. Mod. Phys. 84, 1383 (2012).

26. A. Chubukov, Annu. Rev. Condens. Matter Phys. 3, 57 (2012).

27. E. Dagotto, Rev. Mod. Phys. 66, 763 (1994).

28. D.J. Singh, A.S. Sefat, M.A. McGuire, B.C. Sales, D. Mandrus, L.H. VanBebber, and V. Keppens, Phys. Rev. B 79, 094429 (2009). 
29. Y.-Z. Zhang, I. Opahle, H.O. Jeschke, and R. Valentí, Phys. Rev. B 81, 094505 (2010).

30. J. Knolle, I. Eremin, A.V. Chubukov, and R. Moessner, Phys. Rev. B 81,140506 (2010).

31. D.J. Singh and M.H. Du, Phys. Rev. Lett. 100, 237003 (2008).

32. B. Schmidt, M. Siahatgar, and P. Thalmeier, Phys. Rev. B 81, 165101 (2010).

33. C. Fang, B. Xu, P.C. Dai, T. Xiang, and J.P. Hu, Phys. Rev. B 85, 134406 (2012).

34. F.J. Ma, W. Ji, J.P. Hu, Z.Y. Lu, T. Xiang, Phys. Rev. Lett. 102, 177003 (2009).

35. S. Ducatman, N.B. Perkins, and A. Chubukov, Phys. Rev. Lett. 109,157206 (2012).

36. M. Wang, C. Fang, D.X. Yao, G.T. Tan, L.W. Harriger, Y. Song, T. Netherton, C. Zhang, M. Wang, M.B. Stone, W. Tian, J. Hu, and P. Dai, Nat. Comm. 2, 580 (2011).

37. J. Zhao, D.T. Adroja, D.X. Yao, R. Bewley, S. Li, X.F. Wang, G.Wu, X.H. Chen, J. Hu, and P. Dai, Nat. Phys. 5, 555 (2009).

38. W. Lv, F. Krüger, and P. Phillips, Phys. Rev. B 82, 045125 (2010).

39. D. Stanek, O.P. Sushkov, and G.S. Uhrig, Phys. Rev. B 84, 064505 (2011).

40. J. Hu and H. Ding, Sci. Rep. 2, 381 (2012).

41. J. Fink, S. Thirupathaiah, R. Ovsyannikov, H.A. Dürr, R. Follath, Y. Huang, S. de Jong, M.S. Golden, Y.-Z. Zhang, H.O. Jeschke, R. Valentí, C. Felser, S.D. Farahani, M. Rotter, and D. Johrendt, Phys. Rev. B 79, 155118 (2009).

42. D.J. Singh, Phys. Rev. B 78, 094511 (2008).

43. A. Subedi, L. Zhang, D.J. Singh, and M.H. Du, Phys. Rev. B 78, 134514 (2008).

44. S.O. Diallo, V.P. Antropov, T.G. Perring, C. Broholm, J.J. Pulikkotil, N. Ni, S.L. Bud'ko, P.C. Canfield, A. Kreyssig, A.I. Goldman, and R.J. McQueeney, Phys. Rev. Lett. 102, 187206 (2009).

45. D.K. Pratt, M.G. Kim, A. Kreyssig, Y.B. Lee, G.S. Tucker, A. Thaler, W. Tian, J.L. Zarestky, S.L. Bud'ko, P.C. Canfield, B.N. Harmon, A.I. Goldman, and R.J. McQueeney, Phys. Rev. Lett. 106, 257001 (2011).

46. F. Bondino, E. Magnano, M. Malvestuto, F. Parmigiani, M.A. McGuire, A.S. Sefat, B.C. Sales, R. Jin, D. Mandrus, E.W. Plummer, D.J. Singh, and N. Mannella, Phys. Rev. Lett. 101, 267001 (2008).

47. Z.G. Chen, T. Dong, R.H. Ruan, B.F. Hu, B. Cheng, W.Z. Hu, P. Zheng, Z. Fang, X. Dai, and N.L. Wang, Phys. Rev. Lett. 105, 097003 (2010).

48. A.A. Kordyuk, Fiz. Nizk. Temp. 38, 1119 (2012) [Low Temp. Phys. 38, 888 (2012)].

49. R. Yu and Q.M. Si, Phys. Rev. Lett. 110, 146402 (2013).

50. Y.Z. You and Z.Y. Weng, arXiv:1306.3740v1 (2013).

51. S.L. Yu, J. Guo, and J.X. Li, arXiv:1305.1090v1 (2013).

52. W.G. Yin, C.C. Lee, and W. Ku, Phys. Rev. Lett. 105, 107004 (2010).

53. S.-P. Kou, T. Li, and Z.-Y. Weng, Europhys. Lett. 88, 17010 (2009).
54. Y.-Z. You, F. Yang, S.-P. Kou, and Z.-Y. Weng, Phys. Rev. B 84, 054527 (2011).

55. Y.-Z. You, F. Yang, S.-P. Kou, and Z.-Y. Weng, Phys. Rev. Lett. 107, 167001 (2011).

56. A. Hackl and M. Vojta, New J. Phys. 11, 055064 (2009).

57. M. Yi, D.H. Lu, R. Yu, S.C. Riggs, J.H. Chu, B. Lv, Z.K. Liu, M. Lu, Y.T. Cui, M. Hashimoto, S.K. Mo, Z. Hussain, C.W. Chu, I.R. Fisher, Q. Si, and Z.X. Shen, Phys. Rev. Lett. 110, 067003 (2013).

58. Z.P. Yin, K. Haule, and G. Kotliar, Nature Materials 10, 932 (2011).

59. J. Ferber, K. Foyevtsova, R. Valentí, and H.O. Jeschke, Phys. Rev. B 85, 094505 (2012).

60. Y. Xiao, Y. Su, R. Mittal, T. Chatterji, T. Hansen, C.M.N. Kumar, S. Matsuishi, Y. Inoue, H. Hosono, and Th. Brueckel, Phys. Rev. B 79, 060504 (2009).

61. Y. Xiao, Y. Su, R. Mittal, T. Chatterji, T. Hansen, S. Price, C.M.N. Kumar, J. Persson, S. Matsuishi, Y. Inoue, H. Hosono, Th. Brueckel, Phys. Rev. B 81, 094523 (2010).

62. Y. Zhang, L.X. Yang, M. Xu, Z.R. Ye, F. Chen, C. He, H.C. Xu, J. Jiang, B.P. Xie, J.J. Ying, X.F. Wang, X.H. Chen, J.P. $\mathrm{Hu}$, M. Matsunami, S. Kimura, and D.L. Feng, Nature Materials 10, 273 (2011).

63. W. Li, H. Ding, P. Deng, K. Chang, C.L. Song, K. He, L.L. Wang, X.C. Ma, J.P. Hu, X. Chen, and Q.K. Xue, Nature Physics 8, 126 (2012)

64. S. Avci, O. Chmaissem, D.Y. Chung, S. Rosenkranz, E.A. Goremychkin, J.P. Castellan, I.S. Todorov, J.A. Schlueter, H. Claus, A. Daoud-Aladine, D.D. Khalyavin, M.G. Kanatzidis, and R. Osborn, Phys. Rev. B 85, 184507 (2012).

65. A.K. Maziopa, Z. Shermadini, E. Pomjakushina, V. Pomjakushin, M. Bendele, A. Amato, R. Khasanov, H. Luetkens, and K. Conder, J. Phys: Condens. Matter 23, 052203 (2011).

66. M.H. Fang, H.D. Wang, C.H. Dong, Z.J. Li, C.M. Feng, J. Chen, and H.Q. Yuan, Europhys. Lett. 94, 27009 (2011).

67. F. Ye, S. Chi, W. Bao, X.F. Wang, J.J. Ying, X.H. Chen, H.D. Wang, C.H. Dong, and M.H. Fang, Phys. Rev. Lett. 107, 137003 (2011).

68. C.H. Lee, K. Kihou, H.K. Furukawa, T. Saito, A. Iyo, H. Eisaki, H. Fukazawa, Y. Kohori, K. Suzuki, H. Usui, K. Kuroki, and K. Yamada, Phys. Rev. Lett. 106, 067003 (2011).

69. G.R. Stewart, Rev. Mod. Phys. 83, 1589 (2011).

70. A.S. Sefat, Rep. Prog. Phys. 74, 124502 (2011).

71. T.M. McQueen, M. Regulacio, A.J. Williams, Q. Huang, J.W. Lynn, Y.S. Hor, D.V. West, M.A. Green, and R.J. Cava, Phys. Rev. B 78, 024521 (2008).

72. Z. Deng, X.C. Wang, Q.Q. Liu, S.J. Zhang, Y.X. Lv, J.L. Zhu, R.C. Yu, and C.Q. Jin, Europhys. Lett. 87, 37004 (2009).

73. H. Shishido, A.F. Bangura, A.I. Coldea, S. Tonegawa, K. Hashimoto, S. Kasahara, P.M.C. Rourke, H. Ikeda, T. Terashima, R. Settai, Y. Ōnuki, D. Vignolles, C. Proust, B. Vignolle, A. McCollam, Y. Matsuda, T. Shibauchi, and A. Carrington, Phys. Rev. Lett. 104, 057008 (2010).

74. S.A.J. Kimber, D.N. Argyriou, F. Yokaichiya, K. Habicht, S. Gerischer, T. Hansen, T. Chatterji, R. Klingeler, C. Hess, 
G. Behr, A. Kondrat, and B. Büchner, Phys. Rev. B 78, 140503 (2008).

75. C. de la Cruz, Q. Huang, J.W. Lynn, J.Y. Li, W. Ratcliff, J.L. Zarestky, H.A. Mook, G.F. Chen, J.L. Luo, N.L. Wang, and P.C. Dai, Nature 453, 07057 (2008).

76. Y. Chen, J.W. Lynn, J. Li, G. Li, G.F. Chen, J.L. Luo, N.L. Wang, P.C. Dai, C. de la Cruz, and H.A. Mook, Phys. Rev. B 78, 064515 (2008).

77. J. Zhao, Q. Huang, C. de la Cruz, S.L. Li, J.W. Lynn, Y. Chen, M.A. Green, G.F. Chen, G. Li, Z. Li, J.L. Luo, N.L. Wang, and P.C. Dai, Nature Materials 7, 953 (2008).

78. Y. Qiu, W. Bao, Q. Huang, T. Yildirim, J.M. Simmons, M.A. Green, J.W. Lynn, Y.C. Gasparovic, J. Li, T. Wu, G. Wu, and X.H. Chen, Phys. Rev. Lett. 101, 257002 (2008).

79. C.R. Rotundu, D.T. Keane, B. Freelon, S.D. Wilson, A. Kim, P.N. Valdivia, E. Bourret-Courchesne, and R.J. Birgeneau, Phys. Rev. B 80, 144517 (2009).

80. H. Luetkens, H.H. Klauss, M. Kraken, F.J. Litterst, T. Dellmann, R. Klingeler, C. Hess, R. Khasanov, A. Amato, C. Baines, M. Kosmala, O.J. Schumann, M. Braden, J. HamannBorrero, N. Leps, A. Kondrat, G. Behr, J. Werner, and B. Büchner, Nature Materials 8, 305 (2009).

81. H. Takahashi, H. Okada, K. Igawa, Y. Kamihara, M. Hirano, H. Hosono, K. Matsubayashi, and Y. Uwatoko, J. Supercond. Nov. Magn. 22, 595 (2009).

82. Y. Su, P. Link, A. Schneidewind, Th. Wolf, P. Adelmann, Y. Xiao, M. Meven, R. Mittal, M. Rotter, D. Johrendt, Th. Brueckel, and M. Loewenhaupt, Phys. Rev. B 79, 064504 (2009).

83. J. Zhao, W. Ratcliff, J.W. Lynn, G.F. Chen, J.L. Luo, N.L. Wang, J.P. Hu, and P.C. Dai, Phys. Rev. B 78, 140504 (2008).

84. A.I. Goldman, D.N. Argyriou, B. Ouladdiaf, T. Chatterji, A. Kreyssig, S. Nandi, N. Ni, S.L. Bud'ko, P.C. Canfield, and R.J. McQueeney, Phys. Rev. B 78, 100506 (2008).

85. S. Kasahara, T. Shibauchi, K. Hashimoto, K. Ikada, S. Tonegawa, R. Okazaki, H. Shishido, H. Ikeda, H. Takeya, K. Hirata, T. Terashima, and Y. Matsuda, Phys. Rev. B 81, 184519 (2010).

86. D. Johrendt and R. Pöttgen, Physica C 469, 332 (2009).

87. H. Chen, Y. Ren, Y. Qiu, W. Bao, R.H. Liu, G. Wu, T. Wu, Y.L. Xie, X.F. Wang, Q. Huang, and X.H. Chen, Europhys. Lett. 85, 17006 (2009).

88. S. Nandi, M.G. Kim, A. Kreyssig, R.M. Fernandes, D.K. Pratt, A. Thaler, N. Ni, S.L. Bud'ko, P.C. Canfield, J. Schmalian, R.J. McQueeney, and A.I. Goldman, Phys. Rev. Lett. 104, 057006 (2010).

89. M.G. Kim, D.K. Pratt, G.E. Rustan, W. Tian, J.L. Zarestky, A. Thaler, S.L. Bud'ko, P.C. Canfield, R.J. McQueeney, A. Kreyssig, and A.I. Goldman, Phys. Rev. B 83, 054514 (2011).

90. P.C. Canfield, S.L. Bud'ko, Ni Ni, J.Q. Yan, and A. Kracher, Phys. Rev. B 80, 060501 (2009).

91. X.Y. Lu, H. Gretarsson, R. Zhang, X.R. Liu, H.Q. Luo, W. Tian, M. Laver, Z. Yamani, Y.J. Kim, A.H. Nevidomskyy, Q.M. Si, and P.C. Dai, Phys. Rev. Lett. 110, 257001 (2013).

92. A. Mani, N. Ghosh, S. Paulraj, A. Bharathi, and C.S. Sundar, Europhys. Lett. 87, 17004 (2009).
93. S.A.J. Kimber, A. Kreyssig, Y.-Z. Zhang, H.O. Jeschke, R. Valentí, F. Yokaichiya, E. Colombier, J.Q. Yan, T.C. Hansen, T. Chatterji, R.J. McQueeney, P.C. Canfield, A.I. Goldman, and D.N. Argyriou, Nature Materials 8, 471 (2009).

94. A.E. Taylor, M.J. Pitcher, R.A. Ewings, T.G. Perring, S.J. Clarke, A.T. Boothroyd, Phys. Rev. B 83, 220514 (2011).

95. L. Ma, J. Zhang, G.F. Chen, and W.Q. Yu, Phys. Rev. B 82, 180501 (2010).

96. S.L. Li, C. de la Cruz, Q. Huang, G.F. Chen, T.L. Xia, J.L. Luo, N.L. Wang, and P.C. Dai, Phys. Rev. B 80, 020504 (2009).

97. K. Sasmal, B. Lv, Z.J. Tang, F. Chen, Y.Y. Xue, B. Lorenz, A.M. Guloy, and C.W. Chu, Phys. Rev. B 79, 184516 (2009).

98. G.F. Chen, W.Z. Hu, J.L. Luo, and N.L. Wang, Phys. Rev. Lett. 102, 227004 (2009).

99. D.R. Parker, M.J.P. Smith, T. Lancaster, A.J. Steele, I. Franke, P.J. Baker, F.L. Pratt, M.J. Pitcher, S.J. Blundell, and S.J. Clarke, Phys. Rev. Lett. 104, 057007 (2010).

100. W. Bao, Y. Qiu, Q. Huang, M.A. Green, P. Zajdel, M.R. Fitzsimmons, M. Zhernenkov, S. Chang, M.H. Fang, B. Qian, E.K. Vehstedt, J.H. Yang, H.M. Pham, L. Spinu, and Z.Q. Mao, Phys. Rev. Lett. 102, 247001 (2009).

101. S. Rößler, D. Cherian, W. Lorenz, M. Doerr, C. Koz, C. Curfs, Y. Prots, U.K. Rößler, U. Schwarz, S. Elizabeth, and S. Wirth, Phys. Rev. B 84, 174506 (2011).

102. E.E. Rodriguez, C. Stock, P. Zajdel, K.L. Krycka, C.F. Majkrzak, P. Zavalij, and M.A. Green, Phys. Rev. B 84, 064403 (2011).

103. S.L. Li, C. de la Cruz, Q. Huang, Y. Chen, J.W. Lynn, J.P. Hu, Y.L. Huang, F.C. Hsu, K.W. Yeh, M.K. Wu, and P.C. Dai, Phys. Rev. B 79, 054503 (2009).

104. Y. Xia, D. Qian, L. Wray, D. Hsieh, G.F. Chen, J.L. Luo, N.L. Wang, and M.Z. Hasan, Phys. Rev. Lett. 103, 037002 (2009).

105. M. Bendele, A. Maisuradze, B. Roessli, S.N. Gvasaliya, E. Pomjakushina, S. Weyeneth, K. Conder, H. Keller, and R. Khasanov, Phys. Rev. B 87, 060409 (2013).

106. T.M. McQueen, A.J. Williams, P.W. Stephens, J. Tao, Y. Zhu, V. Ksenofontov, F. Casper, C. Felser, and R.J. Cava, Phys. Rev. Lett. 103, 057002 (2009).

107. S. Margadonna, Y. Takabayashi, Y. Ohishi, Y. Mizuguchi, Y. Takano, T. Kagayama, T. Nakagawa, M. Takata, and K. Prassides, Phys. Rev. B 80, 064506 (2009).

108. T.J. Liu, J. Hu, B. Qian, D. Fobes, Z.Q. Mao, W. Bao, M. Reehuis, S.A.J. Kimber, K. Prokeš, S. Matas, D.N. Argyriou, A. Hiess, A. Rotaru, H. Pham, L. Spinu, Y. Qiu, V. Thampy, A.T. Savici, J.A. Rodriguez, and C. Broholm, Nature Materials 9, 716 (2010).

109. W. Li, H. Ding, Z. Li, P. Deng, K. Chang, K. He, S.H. Ji, L.L. Wang, X.C. Ma, J.P. Hu, X. Chen, and Q.K. Xue, Phys. Rev. Lett. 109, 057003 (2012).

110. H. Lee, Y.-Z. Zhang, H.O. Jeschke, and R. Valentí, Phys. Rev. B 81, 220506 (2010).

111. M.-C. Ding, H.-Q. Lin, and Y.-Z. Zhang, Phys. Rev. B 87, 125129 (2013). 
112. Y.-Z. Zhang, H.C. Kandpal, I. Opahle, H.O. Jeschke, and R. Valentí, Phys. Rev. B 80, 094530 (2009).

113. Y.-Z. Zhang, H. Lee, I. Opahle, H.O. Jeschke, and R. Valentí, J. Phys. Chem. Solids 72, 324 (2011).

114. I. Opahle, H.C. Kandpal, Y.-Z. Zhang, C. Gros, and R. Valent, Phys. Rev. B 79, 024509 (2009).

115. J. Ferber, Y.-Z. Zhang, H.O. Jeschke, and R. Valent, Phys. Rev. B 82, 165102 (2010).

116. Y. Yao, Y.-Z. Zhang, H. Lee, H.O. Jeschke, R. Valent, H.-Q. Lin, C.-Q. Wu, Mod. Phys. Lett. B 27, 1330015 (2013).

117. A. Georges, G. Kotliar, W. Krauth, and M.J. Rozenberg, Rev. Mod. Phys. 68, 13 (1996).

118. E. Gull, A.J. Millis, A.I. Lichtenstein, A.N. Rubtsov, M. Troyer, and P. Werner, Rev. Mod. Phys. 83, 349 (2011).

119. Y.-Z. Zhang, H. Lee, H.-Q. Lin, C.-Q. Wu, H.O. Jeschke, and R. Valent, Phys. Rev. B 85, 035123 (2012).

120. S. Graser, T.A. Maier, P.J. Hirschfeld, and D.J. Scalapino, New J. Phys. 11, 025016 (2009).
121. A.A. Mostofi, J.R. Yates, Y.-S. Lee, I. Souza, D. Vanderbilt, and N. Marzari, Comput. Phys. Commun. 178, 658 (2008).

122. J. Kunes, R. Arita, P. Wissgott, A. Toschi, H. Ikeda, and K. Held, Comput. Phys. Commun. 181, 1888 (2010).

123. P. Blaha, K. Schwarz, G. Madsen, D. Kvaniscka, and J. Luitz, in WIEN2K, An Augmented Plane Wave + Local Orbitals Program for Calculating Crystal, K. Schwarz (ed.), Techn. University, Vienna, Austria (2001).

124. R. Car and M. Parrinello, Phys. Rev. Lett. 55, 2471 (1985).

125. P.E. Blöchl, Phys. Rev. B 50, 17953 (1994).

126. M. Parrinello and A. Rahman, Phys. Rev. Lett. 45, 1196 (1980).

127. S. Thirupathaiah, S. de Jong, R. Ovsyannikov, H.A. Dürr, A. Varykhalov, R. Follath, Y. Huang, R. Huisman, M.S. Golden, Y.-Z. Zhang, H.O. Jeschke, R. Valent, A. Erb, A. Gloskovskii, and J. Fink, Phys. Rev. B 81, 104512 (2010).

128. T. Moriya, K. Ueda, Rep. Prog. Phys. 66, 1299 (2003). 